\title{
Prognostic value of CT-derived myocardial blood flow, CT fractional flow reserve and high-risk plaque features for predicting major adverse cardiac events
}

\author{
Lihua Yu ${ }^{1 \#}$, Zhigang Lu ${ }^{2 \#}$, Xu Dai ${ }^{1}$, Chengxing Shen ${ }^{2}$, Lei Zhang ${ }^{3}$, Jiayin Zhang ${ }^{3}$ \\ ${ }^{1}$ Institute of Diagnostic and Interventional Radiology, Shanghai Jiao Tong University Affiliated Sixth People's Hospital, Shanghai, China; \\ ${ }^{2}$ Department of Cardiology, Shanghai Jiao Tong University Affiliated Sixth People's Hospital, Shanghai, China; ${ }^{3}$ Department of Radiology, Shanghai \\ General Hospital, Shanghai Jiao Tong University School of Medicine, Shanghai, China \\ \#These authors contributed equally to this work. \\ Correspondence to: Jiayin Zhang, MD; Lei Zhang, MD. Department of Radiology, Shanghai General Hospital, Shanghai Jiao Tong University School \\ of Medicine, \#85 Wujin Rd, Shanghai, China. Email: andrewssmu@msn.com; zhanglei4302@hotmail.com.
}

Background: Myocardial blood flow (MBF), CT fractional flow reserve (CT-FFR) and high-risk plaque (HRP) features have been revealed to be associated with patients' prognosis. However, direct intraindividual comparison of these CT-derived parameters has not been explored yet. The aim of this study was to investigate the prognostic value of CT-derived MBF, CT-FFR and HRP features for predicting major adverse cardiac events (MACEs).

Methods: Consecutive patients with chest pain and intermediate-to-high pre-test probability of coronary artery disease (CAD) were prospectively enrolled. All patients were referred for dynamic CT myocardial perfusion imaging (CT-MPI) + coronary CT angiography (CCTA) and followed up for at least 1 year. $\mathrm{MBF}_{\text {ischemic }}$ (mean MBF of all ischemic segments), $\mathrm{MBF}_{\text {ratio }}$ (MBF of ischemic segments/MBF of reference segments), CT-FFR and HRP features were measured and multivariate analysis was used to evaluate the predictive value of all above parameters for MACEs.

Results: One hundred and forty-two patients were included into final analysis. $\mathrm{MBF}_{\text {ischemic }}$ and $\mathrm{MBF}_{\text {ratio }}$ was significantly lower in patients with MACE compared to patients without MACE (87 vs. $153 \mathrm{~mL} / 100 \mathrm{~mL} /$ min and 0.64 vs. 0.95 , both $\mathrm{P}<0.001)$. Similarly, CT-FFR was also markedly lower in patients with MACE (0.58 vs. $0.88, \mathrm{P}<0.001)$ whereas coronary artery calcium score (CACS) was significantly higher $(1,038.9$ vs. 34.2, $\mathrm{P}<0.001)$. According to ROC curve analysis, $\mathrm{MBF}_{\text {ischemic }}, \mathrm{MBF}_{\text {ratio }}$ and $\mathrm{CACS}$ had largest area under curve $(\mathrm{AUC}=0.872,0.855$ and 0.813 respectively, all $\mathrm{P}<0.001)$ for identifying patients with MACE. After adjusted by multivariate analysis, $\mathrm{MBF}_{\text {ischemic }}$ (hazard ratio $=23.382, \mathrm{P}=0.003$ ) and CACS (hazard ratio =3.759, $\mathrm{P}=0.029)$ were revealed to be the independent predictors for MACE where CT-FFR and HRP features failed to have prognostic value.

Conclusions: $\mathrm{MBF}_{\text {ischemic }}$ derived from dynamic CT-MPI was the strongest predictor for MACE, followed by CACS. $\mathrm{MBF}_{\text {ischemic }}$ outperformed HRP features and CT-FFR for prediction of unfavorable clinical outcome.

Keywords: Coronary artery disease (CAD); fractional flow reserve (FFR); myocardial perfusion imaging (MPI); computed tomography (CT); plaque

Submitted Apr 08, 2021. Accepted for publication Jul 11, 2021.

doi: $10.21037 / \mathrm{cdt}-21-219$

View this article at: https://dx.doi.org/10.21037/cdt-21-219 


\section{Introduction}

Dynamic CT myocardial perfusion imaging (CT-MPI) has emerged as one useful approach for absolute quantification of myocardial blood flow (MBF) and consequently precise evaluation of hemodynamic significance of coronary artery disease (CAD) (1-3). Coronary CT angiography (CCTA) in combination with dynamic CT-MPI can provide anatomical and functional assessment to guide treatment strategy (4). In addition, the MBF derived from CT-MPI also bears prognostic value as lower MBF has been found to be related to increased risks of unfavorable clinical outcome (5-7).

Other than MBF, the CCTA data acquired in the integrated dynamic CT-MPI combined with CCTA protocol can also be used to simulate CT fractional flow reserve (CT-FFR) as well as to evaluate high-risk plaque (HRP) features. According to previous studies, both CTFFR and HRP features are associated with major adverse cardiac events (MACE) (8-13).

Although dynamic CT-MPI + CCTA enables "one-stop shop" assessment of all the aforementioned parameters, it remains unclear that which one has the strongest predictive value for clinical outcome. Hence, our purpose was to investigate the prognostic value of MBF, CT-FFR and HRP features for predicting MACE. We hypothesized that MBF had the highest prognostic value for predicting MACE.

We present the following article in accordance with the STARD reporting checklist (available at https://dx.doi. org/10.21037/cdt-21-219).

\section{Methods}

The study was conducted in accordance with the Declaration of Helsinki (as revised in 2013). The study was approved by the ethics committee of Shanghai Jiao Tong University Affiliated Sixth People's Hospital (No. 2016-073) and informed consent was taken from all the patients.

\section{Study population}

From November 1st, 2016 to June 30th 2019, consecutive patients with stable angina or atypical chest pain were prospectively screened and recruited from the cardiology service if the following inclusion criteria were fulfilled: (I) patients were evaluated to have intermediate to high pre-test probability of obstructive CAD according to updated Diamond and Forrester Chest Pain Prediction Rule; (II) patients were at least 18-year-old and without contraindications to the usage of iodine contrast medium and adenosine triphosphate (ATP). The exclusion criteria were: (I) patients with previous history of coronary revascularization; (II) patients with previous history of myocardial infarction; (III) patients with concomitant cardiomyopathy; (IV) poor image quality of CT-MPI or CCTA which were not sufficient for MBF quantification, CT-FFR calculation or plaque analysis; (V) patients underwent early revascularization after index test (within 90 days), (VI) lost follow-up. All patients were followed up for at least 1 year after index test.

\section{Dynamic CT-MPI + CCTA acquisition}

An integrated protocol, which incorporated calcium score, dynamic CT-MPI and CCTA, was employed for acquisition. All patients underwent CT scanning using third generation dual source CT (SOMATOM Force, Siemens Healthineers). In brief, calcium score was calculated to assess the calcification burden of each coronary branch. Before the triggering of dynamic stress CT-MPI acquisition, adenosine triphosphate (ATP) was intravenously infused at the rate of $160 \mu \mathrm{g} / \mathrm{kg} / \mathrm{min}$ for 3 minutes. Dynamic stress CT-MPI acquisition was initiated 4 seconds after the beginning of contrast injection, using a shuttle mode technique. Dynamic stress CT-MPI was triggered at the end-systolic phase $(250 \mathrm{~ms}$ after the $\mathrm{R}$ wave in all patients). Scanning intervals depended on the patient's heart rate and scans were initiated every second or third heart cycle. CARE $\mathrm{kV}$ and CARE Dose 4D were used to reduce radiation dose. The reference tube voltage was $80 \mathrm{kVp}$ and the effective current was $300 \mathrm{mAs}$.

All patients were given nitroglycerin 5 minutes after dynamic stress CT-MPI acquisition. CCTA images were obtained by using prospective ECG-triggered sequential acquisition. The detailed parameters of contrast medium usage, dynamic stress CT-MPI and CCTA scanning were given in Appendix 1.

\section{Image analysis of CT-MPI}

A dedicated kernel (Qr36) was used to reconstruct dynamic stress CT-MPI datasets to reduce the iodine beam-hardening artifacts. Further analysis was performed using a dedicated CT-MPI software package (Myocardial perfusion analysis, VPCT body, Siemens Healthineers, Forchheim, Germany). Motion correction was performed if mis-registration of the left ventricle caused by 
breathing occurred. MBF was calculated using a hybrid deconvolution and maximum slope model model, as previously reported (14).

Based on the 17-segment model with exclusion of apical segment, region of interest (ROI) was manually delineated on short axis view with pseudo color on a segment base to measure the absolute value of MBF (15). When perfusion defect was visually present, the ROIs were drawn separately to cover the whole area of decreased perfusion $\left(\mathrm{MBF}_{\text {ischemic }}\right)$ as well as area without decreased perfusion (reference territory). Stress $\mathrm{MBF}_{\text {ratio }}$ was also measured, which was defined as the mean MBF of decreased perfusion territories versus that of reference territories. In cases with normal myocardial perfusion, $\mathrm{MBF}_{\text {ischemic }}$ was measured as same as that of global $\mathrm{MBF}$ whereas $\mathrm{MBF}_{\text {ratio }}$ was recorded as 1 .

\section{Plaque analysis and CT-FFR simulation}

CCTA datasets were reconstructed with smooth kernel (Bv 40) and third generation iterative reconstruction (IR) technique (strength 3, ADMIRE, Siemens). A dedicated research software (Coronary Plaque Analysis, version 4.3, Siemens Healthineers, Germany) was employed for further analysis of all lesions with stenosis extent $\geq 30 \%$ at any epicardial vessel with diameter $\geq 2 \mathrm{~mm}$. The following parameters and characteristics were measured and recorded: (I) diameter stenosis (DS); (II) HRP features: low-attenuation plaque (LAP) (10), positive remodeling (PR) (10), Napkinring sign (NRS) (11) and spotty calcification (SC) (13). The detailed definitions of the above parameters were given in Appendix 1. HRPs were defined as plaques with at least two HRP features (16). The stenosis severity of individuals was evaluated according to Coronary Artery Disease - Reporting and Data System (CAD-RADS) and patients with CADRADS grade 3 or above were considered the presence of obstructive CAD (16).

Lesion-specific CT-FFR values were measured for all stenosis with DS $\geq 30 \%$ at any epicardial vessel with diameter $\geq 2 \mathrm{~mm}$. The current study used a machine learning (ML)-based approach for CT-FFR simulation (cFFR, version 3.0, Siemens Healthineers, Forchheim, Germany). In brief, this model was trained on a large database of synthesized coronary anatomies, where the reference values are computed using a computational fluid dynamics-based model (17). The details regarding how this ML-based model was trained and how onsite processing was performed were given in Appendix 1. The lesion-specific
CT-FFR values were measured at the distal shoulder of the lesion, where no plaque could be detected. When multiple lesions were present within one patient, the lesion with lowest CT-FFR value was used for further patient-based analysis.

Two experienced radiologists (with 12 years and 3 years of experience of cardiac imaging) who were blinded to clinical histories and outcome independently analyzed all the above parameters and the mean values of quantitative parameters measured by two radiologists were used for further analysis. Disagreements in qualitative parameters between the two radiologists were resolved by discussions to reach a final consensus. The intra-class correlation coefficients (ICC) were used to evaluate the intra-observer and inter-observer agreement of all parameters.

\section{Clinical follow-up and study endpoint}

All enrolled patients were followed up for at least 1 year after dynamic CT-MPI + CCTA by cardiologists who knew the clinical histories and findings of index test via outpatient clinic visit. The primary endpoint of the present study was to determine the predictive value of various CT-derived parameters (perfusion parameters, plaque features and CTFFR) for MACE. MACE was defined as all-cause mortality, myocardial infarction, late revascularization ( $\geq 90$ days after dynamic CT-MPI + CCTA), rehospitalization due to heart failure or aggravated angina symptom.

\section{Statistical analysis}

Statistical analysis was conducted using IBM SPSS Statistics (version 22.0, IBM Corporation, Armonk, NY, USA) and MedCalc Statistical Software (version 11.4.2 MedCalc Software bvba, Ostend, Belgium). Continuous data were represented as mean \pm standard deviation (SD) or median and interquartile range (IQR), depending on whether they were normally distributed (tested with Kolmogorov-Smirnov test). Categorical data were displayed as absolute frequencies and proportions. Interobserver and intra-observer agreement of CT-derived parameters was evaluated by intra-class correlation coefficient (ICC). $t$-test was used for data with normal distribution, while Mann-Whitney $U$ test was used for data that were not normally distributed. The chisquare test or Fisher exact test was used to compare the frequency distribution of categorical and binary data between subgroups, according to the data cell size. 
Receiver operating characteristic curve (ROC) analyses were performed to calculate the AUC of all CT-derived parameters. The optimal cutoff values for coronary artery calcium score (CACS), $\mathrm{MBF}_{\text {ischemic }}$ and $\mathrm{MBF}_{\text {ratio }}$ to predict MACE were calculated using the Youden index whereas preset cutoffs were employed for CAD-RADS $(\geq 3)$, HRP ( $\geq 2$ HRP features) and CT-FFR $(\leq 0.8)$. Diagnostic performance was compared between $\mathrm{MBF}_{\text {ischemic }}$ and other CT-derived parameters by using ROC analysis with the DeLong method. The univariate Cox proportional hazards regression model was used to analyze the prognostic value of CT-derived parameters and clinical characteristics individually for MACE. Multivariate Cox proportional hazards regression analysis was performed to determine the independent predictors of MACE, which was performed using the "forward" method. The model included variables with $\mathrm{P}$ value $<0.20$ in the univariable analysis. Kaplan-Meier survival curves fitted for MACE were used to estimate the cumulative event rates stratified by CT-derived parameters. The log-rank test was used to test for significant differences in cumulative event rates between groups. A two-tail $\mathrm{P}<0.05$ was considered statistically significant.

\section{Results}

\section{Clinical characteristics}

Three hundred and two patients with stable angina and intermediate to high pre-test probability of obstructive were referred for dynamic CT-MPI + CCTA. Among them, 224 patients were initially enrolled according to inclusion and exclusion criteria. Seventy-six patients were excluded due to early revascularization whereas 6 patients were ruled out because of loss of follow-up (details shown in Figure 1). Finally, a total of 142 patients were included in the analysis (mean age $66.66 \pm 12.72$ years, range 37 to 96 years, 96 males). An overview of demographics was presented in Table 1. No adverse events occurred when this index test was performed.

The median dose length product (DLP) of dynamic stress CT-MPI plus CCTA was $372.00 \mathrm{mGy} \times \mathrm{cm}(315.75-$ $471.00 \mathrm{mGy} \times \mathrm{cm}$, range 202.00 to $805.00 \mathrm{mGy} \times \mathrm{cm}$ ), corresponding to $5.21 \mathrm{mSv}(4.42-6.60 \mathrm{mSv}$, range 2.83 to $11.27 \mathrm{mSv}$ ) when using 0.014 as the conversion factor (18). The median amount of contrast material used for dynamic CT-MPI + CCTA was $100 \mathrm{~mL}$ (range 90 to $100 \mathrm{~mL}$ ). There were good Intra-observer and Inter-observer agreements in the measurement of CT-derived parameters (ICC $>0.75$,
$\mathrm{P}<0.001$ for all) (details shown in Tables $\mathrm{S} 1, \mathrm{~S} 2)$.

\section{Clinical outcomes}

The median follow-up time was 23.00 (17.00-32.25) months. In the current cohort, 15 patients $(10.56 \%)$ experienced MACE during the follow-up and met the primary endpoint. Of these 15 patients in whom MACE occurred, 3 patients died from sudden cardiac death, 7 patients underwent late percutaneous coronary intervention, and 5 patients were re-hospitalized due to aggravated angina symptom (4 cases) or heart failure (1 case).

\section{Comparison of CT-derived parameters between patients with and without MACE}

In patients with MACE, $M B F_{\text {ischemic }}$ and $M \mathrm{BF}_{\text {ratio }}$ was significantly lower compared to patients without MACE (87 vs. $153 \mathrm{~mL} / 100 \mathrm{~mL} / \mathrm{min}$ and 0.64 vs. 0.95 , both $\mathrm{P}<0.001$ ) (Figure 2). Similarly, CT-FFR was also markedly lower in patients with MACE (0.58 vs. $0.88, \mathrm{P}<0.001)$ whereas CACS was significantly higher $(1,038.9$ vs. $34.2, \mathrm{P}<0.001)$. Moreover, higher CAD-RADS grades were noted in patients with unfavorable prognosis. In contrast to the above parameters, among HRP features, only PR was more frequently presented in patients with MACE whereas SC, NRS and LAP were found to have similar distribution between two groups (details shown in Table 2, Figure S1).

\section{Prognostic value of CT-derived parameters}

According to ROC curve analysis, $\mathrm{MBF}_{\text {ischemic }}$ had the largest AUC (AUC $=0.872, \mathrm{P}<0.001$ ) among all parameters for identifying patients with MACE, followed by $\mathrm{MBF}_{\text {ratio }}$ and CACS (Figure 3). There was no significant difference between the AUC of $\mathrm{MBF}_{\text {ischemic, }} \mathrm{MBF}_{\text {ratio }}$ and CACS (both $\mathrm{P}>0.05)$ while the AUC of $\mathrm{MBF}_{\text {ischemic }}$ was significantly larger than those of CAD-RADS, HRP and CT-FFR (all $\mathrm{P}<0.05)$.

As revealed by Kaplan-Meier curve analysis, patients with presence of HRPs, CACS $\geq 519.95$, CAD-RADS $\geq 3$, CT-FFR $\leq 0.80, \mathrm{MBF}_{\text {ischemic }} \leq 119.50 \mathrm{~mL} / 100 \mathrm{~mL} / \mathrm{min}$ or $\mathrm{MBF}_{\text {ratio }} \leq 0.82$ were more likely to experience MACE than those without these positive parameters (Figure 4).

Based on univariate Cox proportional hazards regression model, age, diabetes mellitus, presence of HRPs, CACS $\geq 519.95$, CAD-RADS $\geq 3$, CT-FFR $\leq 0.80$, $\mathrm{MBF}_{\text {ischemic }} \leq 119.50 \mathrm{~mL} / 100 \mathrm{~mL} / \mathrm{min}$ and $\mathrm{MBF}_{\text {ratio }} \leq 0.82$ 


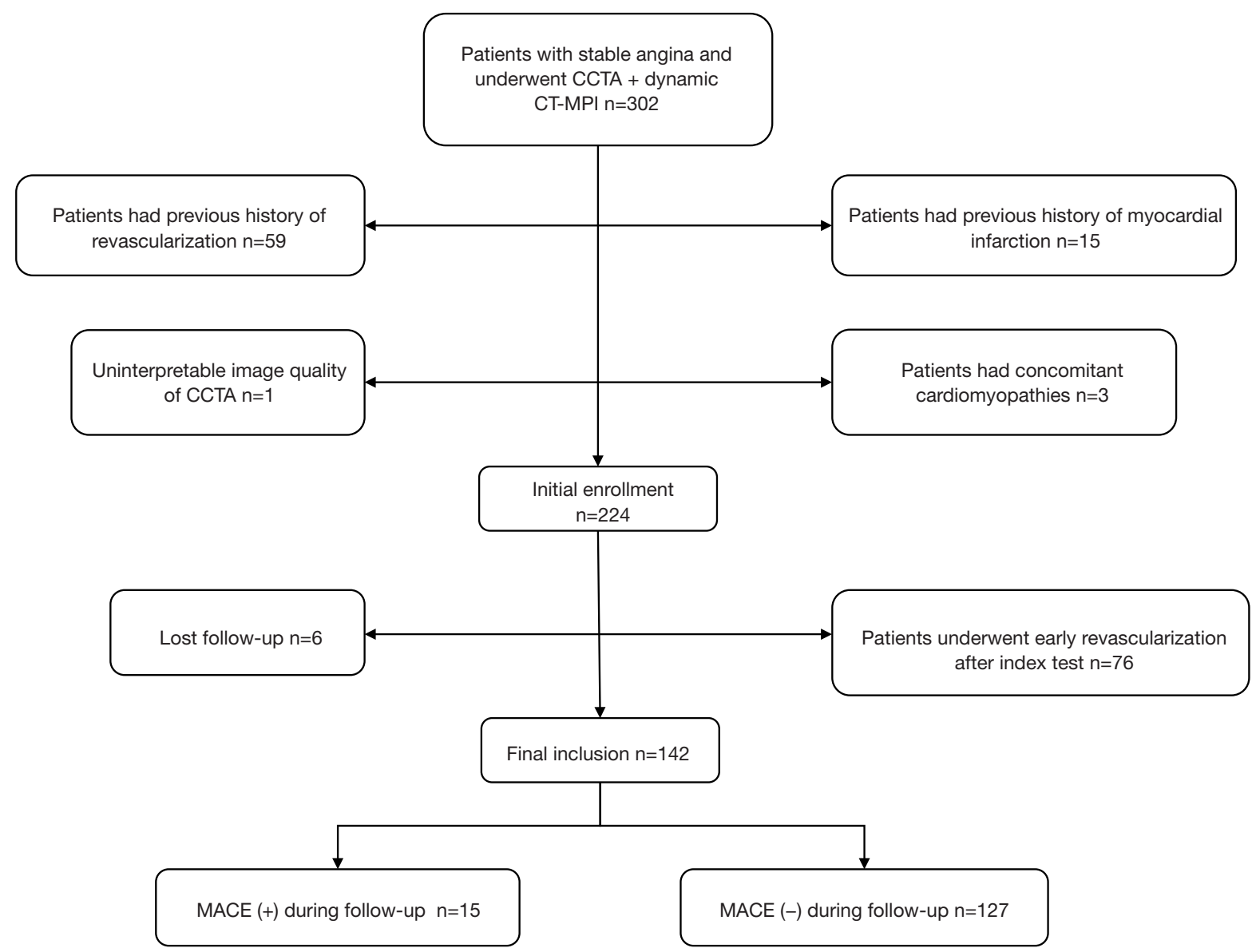

Figure 1 Flow chart of inclusion and exclusion. CCTA, coronary computed tomography angiography; CT-MPI, computed tomography myocardial perfusion imaging; MACE, major adverse cardiac event.

were all significant predictors for MACE. However, after adjusted by multivariate Cox proportional hazards regression model, only $\mathrm{MBF}_{\text {ischemic }}(\mathrm{HR}=23.382, \mathrm{P}=0.003)$ and CACS $(\mathrm{HR}=3.759, \mathrm{P}=0.029)$ remained the independent predictors for MACE (details given in Table 3).

\section{Discussion}

The present study evaluated the prognostic value of various CT-derived parameters and revealed several major findings. First, $\mathrm{MBF}_{\text {ischemic }}$ was found to be the strongest predictor for MACE among all parameters, followed by CACS. Moreover, $\mathrm{MBF}_{\text {ischemic }}$ outperformed HRP and CT-FFR for prediction of unfavorable clinical outcome in the current cohort.

MBF derived from stress dynamic CT-MPI is a useful quantitative parameter representing the global and segmental status of myocardial perfusion. In addition to its discriminative power for the diagnosis of myocardial ischemia (1-3), MBF also bears prognostic value for predicting prognosis (5-7). In the current study, more MACEs occurred in patients with reduced MBF due to late revascularization and rehospitalization for aggravated angina symptom. $\mathrm{MBF}_{\text {ischemic, }}$ which indicated the severity of myocardial ischemia, showed the best predictive power and provided powerful risk estimates of MACE. In contrast, although CT-FFR was another approach for the assessment of hemodynamic significance of coronary stenosis, it failed to be the independent predictor for MACE according to multivariate analysis. This discrepant performance between $\mathrm{MBF}_{\text {ischemic }}$ and CT-FFR could be ascribed to the following reasons. First, the diagnostic accuracy of CT-FFR can be dramatically impaired in the presence of severe vessel calcification $(19,20)$ whereas dynamic CT-MPI is not affected. The overall diagnostic performance of dynamic 
Table 1 Univariate comparison of clinical characteristics between patients with and without MACE

\begin{tabular}{|c|c|c|c|c|}
\hline Characteristics & Total $(n=142)$ & $\operatorname{MACE}(+)(n=15)$ & $\operatorname{MACE}(-)(n=127)$ & $P$ value \\
\hline Males, n (\%) & $96(67.61)$ & $9(60.00)$ & $87(68.50)$ & 0.708 \\
\hline Weight (kg) & $68.00(60.00-74.00)$ & $66.00(58.00-78.00)$ & $68.00(60.00-73.00)$ & 0.757 \\
\hline BMI $\left(\mathrm{kg} / \mathrm{m}^{2}\right)$ & $24.22(22.47-26.02)$ & $24.22(23.23-26.37)$ & $24.22(22.34-25.99)$ & 0.661 \\
\hline \multicolumn{5}{|l|}{ Risk factors, n (\%) } \\
\hline Diabetes mellitus & $41(28.87)$ & 8 (53.33) & $33(25.98)$ & 0.056 \\
\hline Hypertension & 99 (69.72) & $15(100.00)$ & $84(66.14)$ & 0.016 \\
\hline Dyslipidemia & $66(46.48)$ & $6(40.00)$ & $60(47.24)$ & 0.595 \\
\hline Current smoking & 26 (18.31) & $2(13.33)$ & $24(18.90)$ & 0.862 \\
\hline III & $6(4.23)$ & 5 (33.33) & $1(0.79)$ & \\
\hline
\end{tabular}

Values are mean \pm SD, $n(\%)$, or median (IQR). BMI, body mass index; IQR, interquartile range; MACE, major adverse cardiac events; SD, standard deviation.

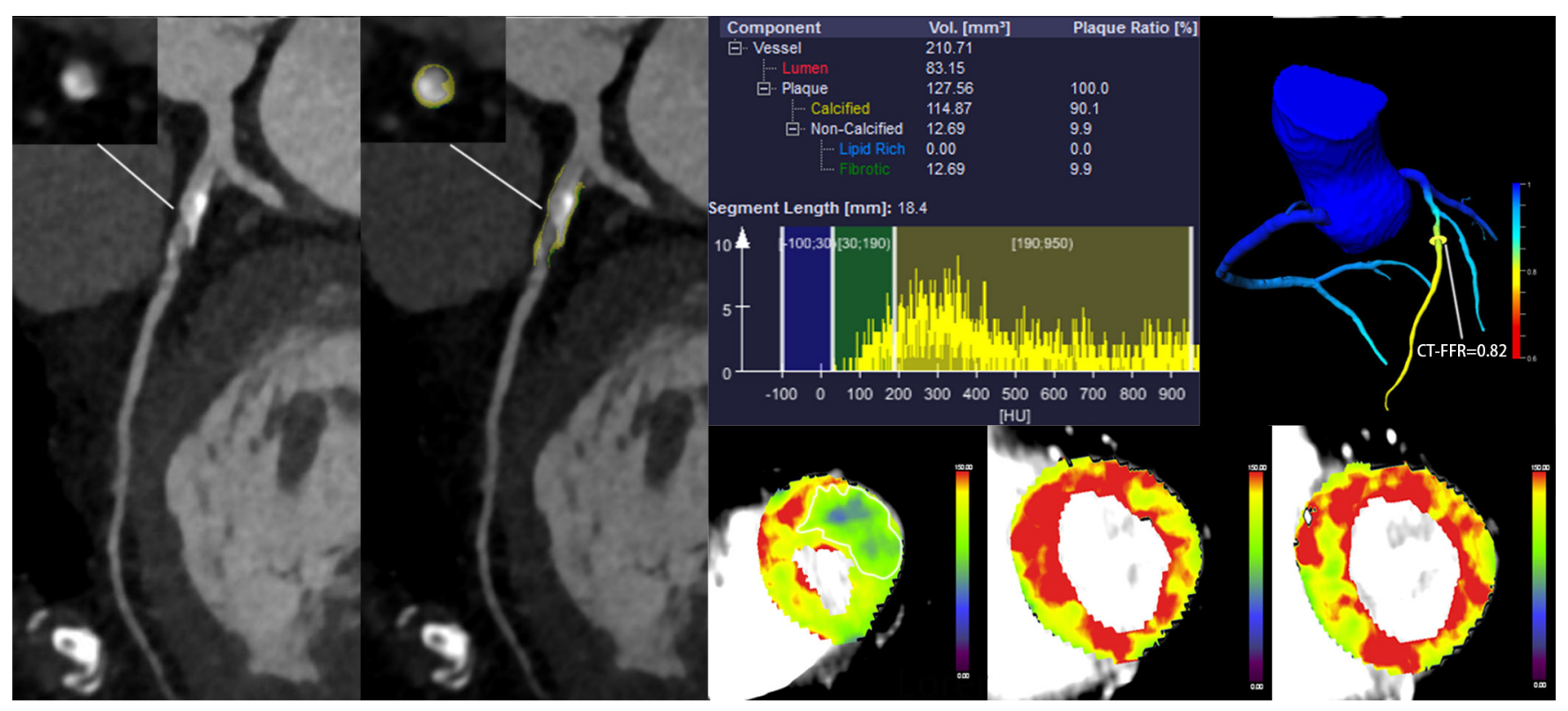

Figure 2 Representative case of patient with MACE. An 86-year-old male with stable angina underwent CCTA + dynamic CT-MPI. CCTA showed mixed plaque with moderate stenosis at proximal LAD. This lesion was revealed to be HRP feature free and hemodynamically insignificant with CT-FFR value of 0.82 . However, dynamic CT-MPI demonstrated decreased myocardial perfusion of apical anterior and apical lateral segments, with $\mathrm{MBF}_{\text {ischemic }}$ of $85 \mathrm{~mL} / 100 \mathrm{~mL} / \mathrm{min}$. This patient underwent late revascularization of LAD lesion due to aggravated angina symptom at follow-up of 27 months after index test. CCTA, coronary computed tomography angiography; CTFFR, computed tomography fractional flow reserve; CT-MPI, computed tomography myocardial perfusion imaging; LAD, left anterior descending; LAP, low attenuation plaque; MACE, major adverse cardiac event; MBF, myocardial blood flow; PR, positive remodeling. 
Table 2 Univariate comparison of CT-derived parameters between patients with and without MACE

\begin{tabular}{|c|c|c|c|c|}
\hline Parameters & Total $(n=142)$ & MACE $(+)(n=15)$ & $\operatorname{MACE}(-)(n=127)$ & $P$ value \\
\hline Napkin-ring sign, n (\%) & $9(6.34)$ & $2(13.33)$ & $7(5.51)$ & 0.243 \\
\hline Positive remodeling, n (\%) & $59(41.55)$ & $10(66.67)$ & 49 (38.58) & 0.037 \\
\hline Low attenuation plaque, n (\%) & $16(11.27)$ & $3(20.00)$ & $13(10.24)$ & 0.484 \\
\hline CAD-RADS classification, $\mathrm{n}(\%)$ & & & & 0.005 \\
\hline$<3$ & $67(47.18)$ & $2(13.33)$ & 65 (51.18) & \\
\hline$\geq 3$ & $75(52.82)$ & $13(86.67)$ & $62(48.82)$ & \\
\hline $\mathrm{HRP}^{\star}, \mathrm{n}(\%)$ & $24(16.90)$ & $5(33.33)$ & $19(14.96)$ & 0.152 \\
\hline CT-FFR & $0.87(0.69-0.95)$ & $0.58(0.50-0.77)$ & $0.88(0.71-0.96)$ & 0.001 \\
\hline
\end{tabular}

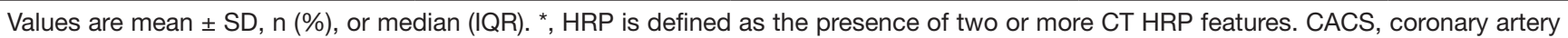
calcium score; CAD-RADS, Coronary Artery Disease - Reporting and Data System; CT= computed tomography; CT-FFR, computed tomography fractional low reserve; IQR, interquartile range; MACE, major adverse cardiac event; MBF, myocardial blood flow; SD, standard deviation.
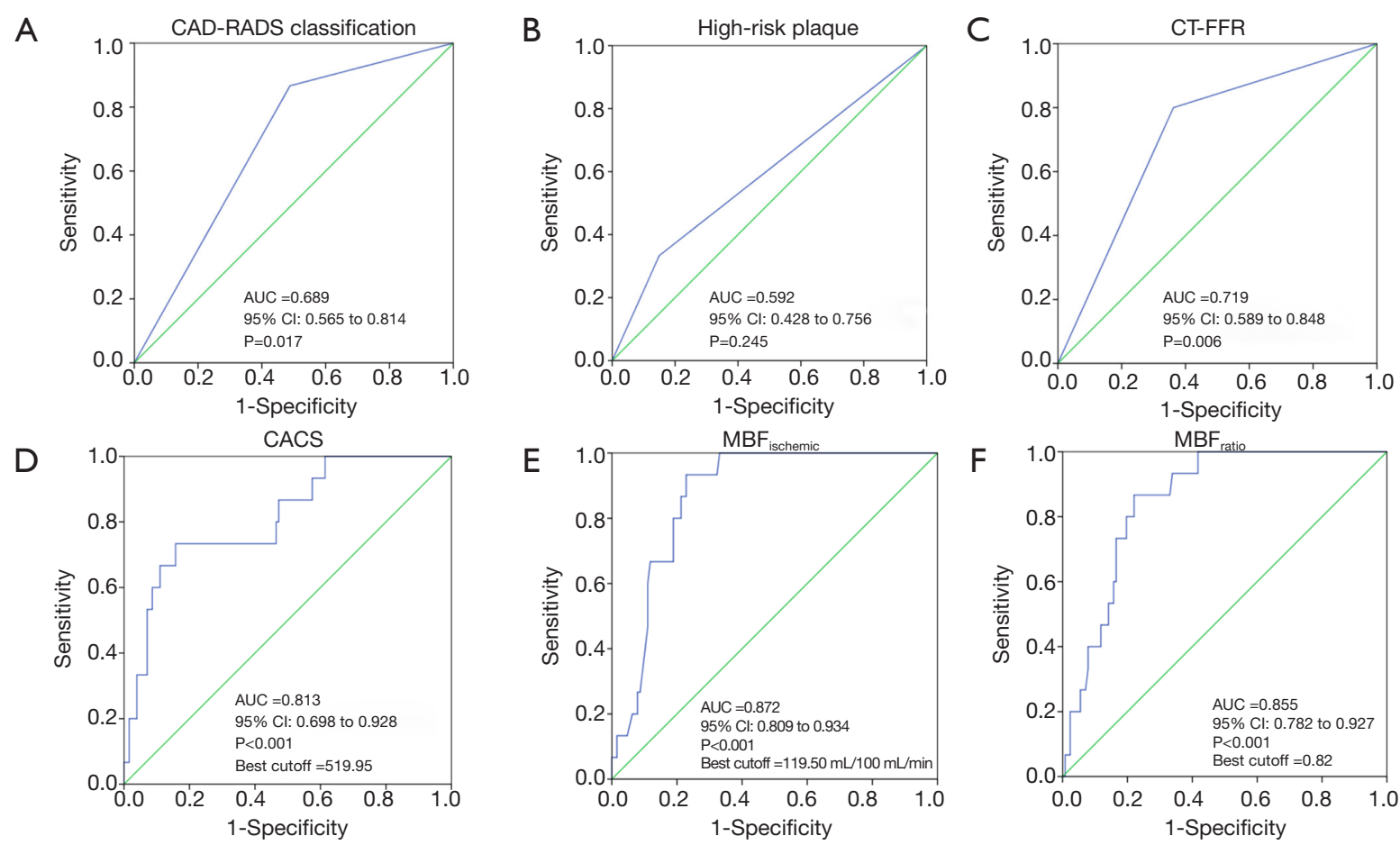

Figure 3 ROC analysis of different parameters for prediction of MACE. For parameters shown in (A,B,C), preset cutoff values (CADRADS $\geq 3$, presence of two or more HRP features, and CT-FFR $\leq 0.8$ ) were used for ROC analysis. For parameters shown in (D,E,F), best cutoff values were generated using the Youden index. AUC , area under curve; CACS, coronary artery calcium score; CAD-RADS, Coronary Artery Disease - Reporting and Data System; CI, confidence interval; CT-FFR, computed tomography fractional flow reserve; MACE, major adverse cardiac event; MBF, myocardial blood flow. 



Figure 4 Kaplan-Meier curves for cumulative event-free rate from MACE according to (A) CAD-RADS classification; (B) HRP; (C) CTFFR; (D) CACS; (E) $\mathrm{MBF}_{\text {ischemic }}$ and (F) $\mathrm{MBF}_{\text {ratio }}$ CACS, coronary artery calcium score; CAD-RADS, Coronary Artery Disease - Reporting and Data System; CT-FFR, computed tomography fractional flow reserve; MACE, major adverse cardiac event; MBF, myocardial blood flow.

CT-MPI outperformed CT-FFR for detection of ischemic coronary stenosis and had less false positive results (1). Second, MBF represents the myocardial perfusion status secondary to the impacts from epicardial vessel disease as well as microvascular dysfunction. In contrast, CTFFR is the parameter only suggesting the hemodynamic significance of epicardial vessel stenosis (21). In light of the above facts, MBF is not only more accurate than CT-FFR for diagnosing myocardial ischemia, but also able to evaluate microvascular dysfunction which is also associated with patients' prognosis (22). Therefore, it is conceivable that MBF outperformed CT-FFR in terms of risk stratification.

Another important finding of the present study demonstrated that MBF was a stronger predictor than HRP with regard to prognostic evaluation, which has not been previously reported. HRP features as assessed by CCTA are thought be the fingerprints for the plaque phenotype that is prone to rupture and associated unfavorable clinical outcome $(23,24)$. Previous studies have confirmed that plaques with large volume and positive vessel remodeling are more unstable than plaques without these features, which is more likely to lead to adverse clinical outcome $(9,10)$. Our study also confirmed that patients with MACE had a higher incidence of positive remodeling than those without MACE. Nevertheless, late revascularization rather than acute coronary syndrome accounted for a large proportion of MACEs occurred in the current cohort. MBF is a parameter for precise evaluation of myocardial ischemia and therefore had larger predictive power for MACEs, which were primarily driven by ischemic coronary stenosis.

It is also of note that CACS was another independent predictor for MACE, which is in line with the results from MESA study (25). As revealed by previous nuclear MPI studies, in patients with CACS $>400$, the overall incidence 
Table 3 Univariate and multivariate analysis: CT-derived parameters and clinical characteristics predicting MACE

\begin{tabular}{|c|c|c|c|c|c|c|}
\hline Variables & \multicolumn{3}{|c|}{ Univariate analysis } & \multicolumn{3}{|c|}{ Multivariate analysis* } \\
\hline CACS $\geq 519.95$ & 11.109 & $3.535-34.916$ & $<0.001$ & 3.759 & $1.146-12.329$ & 0.029 \\
\hline CAD-RADS $\geq 3$ & 7.094 & $1.591-31.619$ & 0.010 & & & \\
\hline HRP & 2.955 & $1.005-8.688$ & 0.049 & & & \\
\hline $\mathrm{MBF}_{\text {ratio }} \leq 0.82$ & 21.550 & $4.819-96.377$ & $<0.001$ & & & \\
\hline CT-FFR $\leq 0.8$ & 6.827 & $1.922-24.253$ & 0.003 & & & \\
\hline Age $\geq 71$ years & 4.855 & $1.545-15.260$ & 0.007 & & & \\
\hline Male & 0.655 & $0.233-1.844$ & 0.424 & & & \\
\hline Dyslipidemia & 0.735 & $0.261-2.066$ & 0.559 & & & \\
\hline Current smoking & 0.773 & $0.174-3.443$ & 0.736 & & & \\
\hline
\end{tabular}

*, data for variables not included in the equation using the "forward" method were not provided. CACS, coronary artery calcium score; CAD-RADS, Coronary Artery Disease - Reporting and Data System; CI, confidence interval; CT, computed tomography; CT-FFR, computed tomography fractional low reserve; HR, hazard ratio; IQR, interquartile range; MACE, major adverse cardiac event; MBF, myocardial blood flow; SD, standard deviation.

of myocardial ischemia was as high as $35.2 \%$ (26). In other words, higher CACS is associated with the presence of decreased MBF, which is more likely to result in revascularization.

The potential clinical implications of the present study lie in the following aspects. First, given the highest prognostic value, $\mathrm{MBF}_{\text {ischemic }}$ derived from dynamic CT-MPI may be the ideal parameter for precise risk stratification in patients with intermediate to high pre-test probability of CAD. The presence of reduced MBF in patients with suspected CAD should raise caution in clinical practice because it was closely associated with late revascularization. Further investigations, such as ICA and invasive FFR, are warranted to optimize the treatment strategy. Second, CACS also has strong predictive power for MACEs according to multivariate analysis so that calcium score scan might serve as an easy-to-perform method for preliminary screening in patients with intermediate to high pre-test probability of obstructive CAD. For patients with high CACS score, dynamic CT-MPI combined with CCTA is more preferred than CCTA alone for comprehensive assessment of disease severity and risk stratification.

Despite the above promising findings, the current study has several limitations. First, the overall MACE number in the present cohort was relatively small and the major type of MACE was late revascularization. This heterogeneous distribution of MACE type might have an impact on the result of predictive value of different parameters. Therefore, future prospective studies with larger sample size and longer follow-up period are warranted to validate the current findings. Moreover, the current cohort referred to the population with stable angina or atypical chest pain and immediate to high pre-test probability of obstructive CAD. More than half of patients had obstructive stenosis (CAD-RADS $\geq 3$ ). Thus, the present findings could not be directly applied to a more general population. In addition, the myocardial perfusion parameters were derived from third generation of dual source CT using a hybrid deconvolution model in the current study. Different calculation algorithms can lead to varied values of quantified MBF (27). Thus, the present cut-off value of $\mathrm{MBF}_{\text {ischemic }}$ could not be directly employed in other cohorts quantified using different calculation algorithms. Finally, the contrast load of CT examination was relatively high due to double injection of contrast medium for CT-MPI and CCTA. This might limit the clinical application in 
patients with impaired renal function.

In conclusion, $\mathrm{MBF}_{\text {ischemic }}$ was found to be the strongest predictor for MACE among all parameters, followed by CACS. Moreover, $\mathrm{MBF}_{\text {ischemic }}$ outperformed HRP and CTFFR for prediction of unfavorable clinical outcome in the current cohort.

\section{Acknowledgments}

Funding: This study is supported by National Natural Science Foundation of China (Grant No. 81671678), Medical Guidance Scientific Research Support Project of Shanghai Science and Technology Commission (Grant No. 19411965100) and Shanghai Municipal Education Commission-Gaofeng Clinical Medicine Grant Support (Grant No. 20161428).

\section{Footnote}

Reporting Checklist: The authors have completed the STARD reporting checklist. Available at https://dx.doi. org/10.21037/cdt-21-219

Data Sharing Statement: Available at https://dx.doi. org/10.21037/cdt-21-219

Conflicts of Interest: All authors have completed the ICMJE uniform disclosure form (available at https://dx.doi. org/10.21037/cdt-21-219). The authors have no conflicts of interest to declare.

Ethical Statement: The authors are accountable for all aspects of the work in ensuring that questions related to the accuracy or integrity of any part of the work are appropriately investigated and resolved. The study was conducted in accordance with the Declaration of Helsinki (as revised in 2013). The study was approved by the ethics committee of Shanghai Jiao Tong University Affiliated Sixth People's Hospital (No. 2016-073) and informed consent was taken from all the patients.

Open Access Statement: This is an Open Access article distributed in accordance with the Creative Commons Attribution-NonCommercial-NoDerivs 4.0 International License (CC BY-NC-ND 4.0), which permits the noncommercial replication and distribution of the article with the strict proviso that no changes or edits are made and the original work is properly cited (including links to both the formal publication through the relevant DOI and the license). See: https://creativecommons.org/licenses/by-nc-nd/4.0/.

\section{References}

1. Li Y, Yu M, Dai X, et al. Detection of Hemodynamically Significant Coronary Stenosis: CT Myocardial Perfusion versus Machine Learning CT Fractional Flow Reserve. Radiology 2019;293:305-14.

2. Yang J, Dou G, He B, et al. Stress Myocardial Blood Flow Ratio by Dynamic CT Perfusion Identifies Hemodynamically Significant CAD. JACC Cardiovasc Imaging 2020;13:966-76.

3. Pontone G, Baggiano A, Andreini D, et al. Dynamic Stress Computed Tomography Perfusion With a Whole-Heart Coverage Scanner in Addition to Coronary Computed Tomography Angiography and Fractional Flow Reserve Computed Tomography Derived. JACC Cardiovasc Imaging 2019;12:2460-71.

4. Yu M, Shen C, Dai X, et al. Clinical Outcomes of Dynamic Computed Tomography Myocardial Perfusion Imaging Combined With Coronary Computed Tomography Angiography Versus Coronary Computed Tomography Angiography-Guided Strategy. Circ Cardiovasc Imaging 2020;13:e009775.

5. Nakamura S, Kitagawa K, Goto Y, et al. Incremental Prognostic Value of Myocardial Blood Flow Quantified With Stress Dynamic Computed Tomography Perfusion Imaging. JACC Cardiovasc Imaging 2019;12:1379-87.

6. Meinel FG, Pugliese F, Schoepf UJ, et al. Prognostic Value of Stress Dynamic Myocardial Perfusion CT in a Multicenter Population With Known or Suspected Coronary Artery Disease. AJR Am J Roentgenol 2017;208:761-9.

7. van Assen M, De Cecco CN, Eid M, et al. Prognostic value of CT myocardial perfusion imaging and CT-derived fractional flow reserve for major adverse cardiac events in patients with coronary artery disease. J Cardiovasc Comput Tomogr 2019;13:26-33.

8. Yu M, Li W, Lu Z, et al. Quantitative baseline CT plaque characterization of unrevascularized non-culprit intermediate coronary stenosis predicts lesion volume progression and long-term prognosis: A serial CT followup study. Int J Cardiol 2018;264:181-6.

9. Yu M, Lu Z, Li W, et al. Coronary plaque characteristics on baseline CT predict the need for late revascularization in symptomatic patients after percutaneous intervention. Eur Radiol 2018;28:3441-53. 
10. Motoyama S, Sarai M, Harigaya H, et al. Computed tomographic angiography characteristics of atherosclerotic plaques subsequently resulting in acute coronary syndrome. J Am Coll Cardiol 2009;54:49-57.

11. Min JK, Shaw LJ, Devereux RB, et al. Prognostic value of multidetector coronary computed tomographic angiography for prediction of all-cause mortality. J Am Coll Cardiol 2007;50:1161-70.

12. Lee JM, Choi G, Koo BK, et al. Identification of HighRisk Plaques Destined to Cause Acute Coronary Syndrome Using Coronary Computed Tomographic Angiography and Computational Fluid Dynamics. JACC Cardiovasc Imaging 2019;12:1032-43.

13. Duguay TM, Tesche C, Vliegenthart R, et al. Coronary Computed Tomographic Angiography-Derived Fractional Flow Reserve Based on Machine Learning for Risk Stratification of Non-Culprit Coronary Narrowings in Patients with Acute Coronary Syndrome. Am J Cardiol 2017;120:1260-6.

14. Bamberg F, Klotz E, Flohr T, et al. Dynamic myocardial stress perfusion imaging using fast dual-source CT with alternating table positions: initial experience. Eur Radiol 2010;20:1168-73.

15. Cerqueira MD, Weissman NJ, Dilsizian V, et al. Standardized myocardial segmentation and nomenclature for tomographic imaging of the heart. A statement for healthcare professionals from the Cardiac Imaging Committee of the Council on Clinical Cardiology of the American Heart Association. Circulation 2002;105:539-42.

16. Cury RC, Abbara S, Achenbach S, et al. CAD-RADS ${ }^{\mathrm{TM}}$ : Coronary Artery Disease - Reporting and Data System: An Expert Consensus Document of the Society of Cardiovascular Computed Tomography (SCCT), the American College of Radiology (ACR) and the North American Society for Cardiovascular Imaging (NASCI). Endorsed by the American College of Cardiology. J Am Coll Radiol 2016;13:1458-1466.e9.

17. Itu L, Rapaka S, Passerini T, et al. A machine-learning approach for computation of fractional flow reserve from coronary computed tomography. J Appl Physiol (1985) 2016;121:42-52.

18. Tan SK, Yeong CH, Ng KH, et al. Recent Update on Radiation Dose Assessment for the State-of-the-Art Coronary Computed Tomography Angiography Protocols. PLoS One 2016;11:e161543.
19. Yu M, Lu Z, Shen C, et al. The best predictor of ischemic coronary stenosis: subtended myocardial volume, machine learning-based FFRCT, or high-risk plaque features? Eur Radiol 2019;29:3647-57.

20. Yu M, Lu Z, Li W, et al. CT morphological index provides incremental value to machine learning based CT-FFR for predicting hemodynamically significant coronary stenosis. Int J Cardiol 2018;265:256-61.

21. Schuijf JD, Ko BS, Di Carli MF, et al. Fractional flow reserve and myocardial perfusion by computed tomography: a guide to clinical application. Eur Heart J Cardiovasc Imaging 2018;19:127-35.

22. Doyle M, Weinberg N, Pohost GM, et al. Prognostic value of global MR myocardial perfusion imaging in women with suspected myocardial ischemia and no obstructive coronary disease: results from the NHLBI-sponsored WISE (Women's Ischemia Syndrome Evaluation) study. JACC Cardiovasc Imaging 2010;3:1030-6.

23. Nerlekar N, Ha FJ, Cheshire C, et al. Computed Tomographic Coronary Angiography-Derived Plaque Characteristics Predict Major Adverse Cardiovascular Events: A Systematic Review and Meta-Analysis. Circ Cardiovasc Imaging 2018;11:e006973.

24. Bom MJ, van der Heijden DJ, Kedhi E, et al. Early Detection and Treatment of the Vulnerable Coronary Plaque: Can We Prevent Acute Coronary Syndromes? Circ Cardiovasc Imaging 2017;10:e005973.

25. Detrano R, Guerci AD, Carr JJ, et al. Coronary calcium as a predictor of coronary events in four racial or ethnic groups. N Engl J Med 2008;358:1336-45.

26. Hacker $M$, Becker $C$. The incremental value of coronary artery calcium scores to myocardial single photon emission computer tomography in risk assessment. J Nucl Cardiol 2011;18:700-11; quiz 712-6.

27. van Assen M, Pelgrim GJ, De Cecco CN, et al. Intermodel disagreement of myocardial blood flow estimation from dynamic CT perfusion imaging. Eur J Radiol 2019;110:175-80.

Cite this article as: Yu L, Lu Z, Dai X, Shen C, Zhang L, Zhang J. Prognostic value of CT-derived myocardial blood flow, CT fractional flow reserve and high-risk plaque features for predicting major adverse cardiac events. Cardiovasc Diagn Ther 2021;11(4):956-966. doi: 10.21037/cdt-21-219 


\section{Dynamic CT-MPI + coronary CT angiography protocol}

Calcium score was calculated to assess the calcification burden of each coronary branch. The scan range of dynamic stress CT-MPI was confirmed on the basis of the images of calcium score, covering the whole left ventricle and all epicardial vessels. Before the triggering of dynamic stress CT-MPI acquisition, adenosine triphosphate was intravenously infused at the rate of $160 \mu \mathrm{g} / \mathrm{kg} / \mathrm{min}$ for 3 minutes. All subjects were injected with a fixed volume of contrast media $(50 \mathrm{~mL}$, Ultravist, $370 \mathrm{mg}$ iodine $/ \mathrm{mL}$, Bayer) at the rate of $6 \mathrm{~mL} / \mathrm{s}$, followed by a $40 \mathrm{~mL}$ saline flush by using dual-barrel power injector (Tyco, Cincinnati, US). Dynamic stress CT-MPI acquisition was initiated $4 \mathrm{~s}$ after the beginning of contrast injection with a coverage of $10.5 \mathrm{~cm}$ for complete imaging of the whole left ventricle, using a shuttle mode technique. Image acquisition was triggered at the end-systolic phase $(250 \mathrm{~ms}$ after the $\mathrm{R}$ wave). Scanning intervals depended on the participant's heart rate and scans were initiated every second or third heart cycle. Data acquisition was performed for $32 \mathrm{~s}$ with a total of 10 to 15 phases. The scan parameters of dynamic stress CT-MPI is listed as follow: gantry rotation time $=250 \mathrm{~ms}$, collimation $=96 \times 0.6 \mathrm{~mm}$, automated tube current modulation (CARE Dose 4D, Siemens Healthineers) was used and the effective current $=300 \mathrm{mAs}$, automated tube voltage modulation (CAREKv, Siemens Healthineers) was used and the reference tube voltage $=80 \mathrm{kVp}$, reconstructed slice thickness $=3 \mathrm{~mm}$ and reconstructed slice interval $=2 \mathrm{~mm}$.

All patients were given nitroglycerin 5 minutes after dynamic stress CT-MPI acquisition, prior to the acquisition of coronary CT angiography. A bolus tracking technique was used in CCTA acquisition and regions of interest was placed in the ascending aorta. A bolus of contrast media was intravenously injected at the rate of $4-5 \mathrm{~mL} / \mathrm{s}$, followed by injection of a $40 \mathrm{ml}$ saline flush by using dual-barrel power injector. The dose of the contrast media was determined based on the patient's body weight (patients with body mass index $<18$ injected with $40 \mathrm{~mL}$ contrast media at $4 \mathrm{~mL} / \mathrm{s}$, patients with body mass index between 18 and 24 injected with $50 \mathrm{~mL}$ contrast media at $4.5 \mathrm{~mL} / \mathrm{s}$, patients with body mass index $>24$ injected with $60 \mathrm{~mL}$ contrast media at $5 \mathrm{~mL} / \mathrm{s}$ ). CCTA images were obtained by using prospective ECG-triggered sequential acquisition, with the acquisition window covering from $35 \%$ to $75 \%$ of R-R interval, with gantry rotation time $=250 \mathrm{~ms}$ collimation $=96 \times 0.6 \mathrm{~mm}$, reconstructed slice thickness $=0.75 \mathrm{~mm}$, reconstructed slice interval $=0.5 \mathrm{~mm}$ and application of CAREKv and CARE Dose 4D. The reference tube current was set as $320 \mathrm{mAs}$ and the reference tube voltage was set as $100 \mathrm{kVp}$.

\section{Plaque analysis and CT-FFR simulation}

CCTA datasets were reconstructed with smooth kernel ( $\mathrm{Bv} 40)$ and third generation iterative reconstruction (IR) technique (strength 3, ADMIRE, Siemens). A dedicated research software (Coronary Plaque Analysis, version 4.3, Siemens Healthineers, Germany) was employed for further analysis of all lesions with stenosis extent $\geq 30 \%$ at any epicardial vessel with diameter $\geq 2 \mathrm{~mm}$. The following indices were measured and recorded: (I) diameter stenosis (DS) was calculated as (reference diameter - minimal lumen diameter)/reference diameter and was measured manually with a digital caliper at the narrowest level of the lesion and the proximal reference on the cross-sectional images; (II) remodeling index was defined as a maximal lesion vessel diameter divided by proximal reference vessel diameter (at the site where no plaque component can be detected), with positive remodeling (PR) defined as a remodeling index $\geq 1.1$; (III) low-attenuation plaque (LAP) was defined as non-calcified plaques with low-density components (CT value <30 HU); (IV) spotty calcification (SC) was defined by an intra-lesion calcific plaque $<3 \mathrm{~mm}$ in length that comprised <90 degrees of the lesion circumference; (V) Napkin-ring sign (NRS) was characterized by a plaque core with low attenuation areas on CT surrounded by a rim-like area of higher attenuation (CT value $\leq 130 \mathrm{HU}$ ) as previously reported. HRPs were defined as plaques with at least two HRP (LAP, PR, NRS and SC) features. The coronary stenosis of individuals was evaluated according to Coronary Artery Disease - Reporting and Data System (CAD-RADS) and patients with CAD-RADS grade 3 or above were considered the presence of obstructive CAD.

Lesion-specific CT-FFR values were measured for all stenosis with DS $\geq 30 \%$ at any epicardial vessel with diameter $\geq 2 \mathrm{~mm}$. The current study used a machine learning (ML)-based approach, which is an alternative to physics-based approach and can be used on-site to simulate CT-FFR (cFFR, version 3.0, Siemens Healthineers, Forchheim, Germany). It's trained using a synthetically generated database of 12,000 different anatomies of coronary arteries with randomly placed stenosis among 
different branches and bifurcations. A computational fluid dynamic (CFD) by solving reduced-ordered Navier-Stokes equations is applied to calculate the pressure and flow distribution for each coronary tree. Quantitative features of anatomy and computed CT-FFR value were extracted for each location along the coronary tree. Then deep machine learning model is trained by using a deep neural network with four hidden layers to learn the relationship between the FFR value and quantitative anatomic features.

For the on-site processing, the centerline and luminal contours for whole coronary tree were automatically generated after CCTA data were successfully loaded. The centerline and luminal contour which can be manually adjusted when needed are fundamental and critical information for computing CT-FFR value. Users need to label all stenotic lesions manually to extract their geometrical features required for cFFR algorithm. Finally, those data were input into the pre-learned model and cFFR at all locations were generated automatically, and the resulting values were visualized by color-coded $3 \mathrm{D}$ coronary maps. The lesion-specific CT-FFR values were measured at the distal shoulder of the lesion, where no plaque could be detected.

Table S1 Intra-observer reproducibility

\begin{tabular}{llll}
\hline Variables & ICC & $95 \% \mathrm{Cl}$ & $\mathrm{P}$ value \\
\hline $\mathrm{DS}(\%)$ & 0.907 & $0.828-0.950$ & $<0.001$ \\
$\mathrm{MBF}_{\text {ischemic }}$ & 0.938 & $0.887-0.967$ & $<0.001$ \\
$\mathrm{MBF}_{\text {ratio }}$ & 0.939 & $0.888-0.967$ & $<.001$ \\
$\mathrm{CT}-\mathrm{FFR}$ & 0.921 & $0.840-0.960$ & $<0.001$ \\
Spotty calcium & 0.884 & $0.827-0.923$ & $<0.001$ \\
Napkin-ring sign & 0.877 & $0.818-0.918$ & $<0.001$ \\
Positive remodeling & 0.911 & $0.844-0.950$ & $<0.001$ \\
Low attenuation plaque & 0.893 & $0.831-0.933<$ & $<0.001<$
\end{tabular}

$\mathrm{Cl}$, confidence interval; ICC, intraclass correlation coefficient; DS, diameter stenosis; CT-FFR, computed tomography fractional flow reserve; MBF, myocardial blood flow.

Table S2 Inter-observer reproducibility

\begin{tabular}{llll}
\hline Variables & ICC & $95 \% \mathrm{Cl}$ & $\mathrm{P}$ value \\
\hline $\mathrm{DS}(\%)$ & 0.913 & $0.842-0.953$ & $<0.001$ \\
$\mathrm{MBF}_{\text {ischemic }}$ & 0.928 & $0.868-0.961$ & $<0.001$ \\
$\mathrm{MBF}_{\text {ratio }}$ & 0.922 & $0.857-0.958$ & $<0.001$ \\
$\mathrm{CT}-\mathrm{FFR}$ & 0.911 & $0.826-0.954$ & $<0.001$ \\
Spotty calcium & 0.824 & $0.745-0.880$ & $<0.001$ \\
Napkin-ring sign & 0.840 & $0.767-0.891$ & $<0.001$ \\
Positive remodeling & 0.873 & $0.783-0.926$ & $<.001$ \\
Low attenuation plaque & 0.857 & $0.782-0.908$ & $<0.001$ \\
\hline
\end{tabular}

$\mathrm{Cl}$, confidence interval; ICC, intraclass correlation coefficient; DS, diameter stenosis; CT-FFR, computed tomography fractional flow reserve; MBF, myocardial blood flow. 


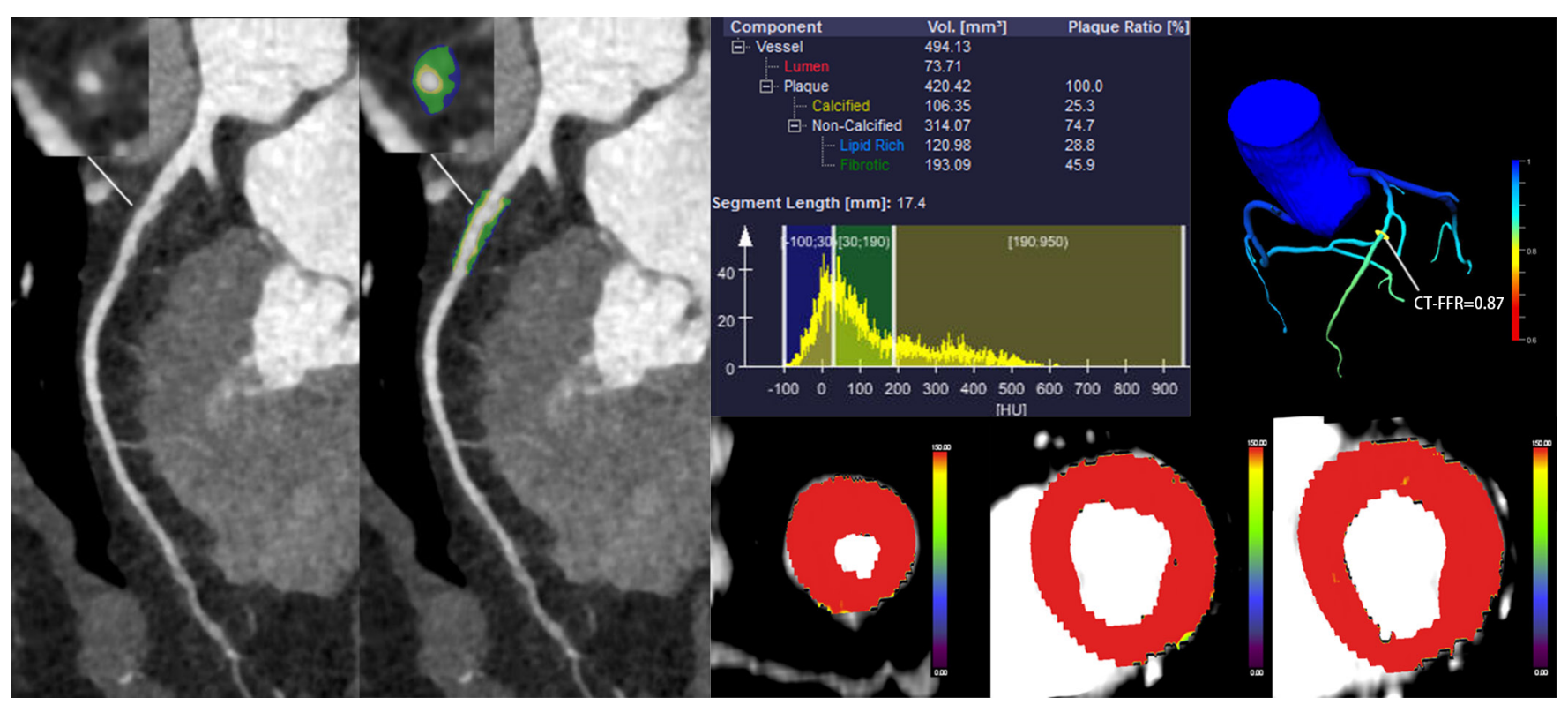

Figure S1 Representative case of patient without MACE. A 69-year-old female with atypical chest pain underwent CCTA + dynamic CT-MPI. CCTA showed mixed plaque with mild stenosis at proximal LAD. This lesion was revealed to have two HRP features (LAP and PR) and hemodynamically insignificant with CT-FFR value of 0.87. Dynamic CT-MPI demonstrated normal myocardial perfusion, with global MBF of $215 \mathrm{~mL} / 100 \mathrm{~mL} / \mathrm{min}$. This patient was MACE free during a follow-up period of 17 months. CCTA, coronary computed tomography angiography; CT-FFR, computed tomography fractional flow reserve; CT-MPI, computed tomography myocardial perfusion imaging; LAD, left anterior descending; LAP, low attenuation plaque; MACE, major adverse cardiac event; MBF, myocardial blood flow; PR, positive remodeling. 